\title{
Diagnóstico de leucemias bifenotípicas por citometría de flujo
}

\author{
Martha Vizcaíno', Carolina Guzmán¹, Iliana De los Reyes, \\ Sandra Quijano 2,3 , Alba Campos² \\ 1 Centro Javeriano de Oncología, Bogotá, D.C., Colombia \\ 2 Hospital Universitario San Ignacio, Bogotá, D.C., Colombia \\ ${ }^{3}$ Facultad de Ciencias, Pontificia Universidad Javeriana, Bogotá, D.C., Colombia \\ Las leucemias bifenotípicas son raras en los niños y representan menos de $5 \%$ de todas las leucemias \\ agudas. El diagnóstico requiere de una evaluación cuidadosa con apoyo de la morfología celular, \\ la citogenética y la citometría de flujo. El tratamiento está orientado a remitir el clon predominante \\ de células, aunque no existen datos conclusivos sobre la remisión completa. En el tratamiento, la \\ quimioterapia ha demostrado mejores resultados que el trasplante de médula ósea. La supervivencia \\ está influenciada por la respuesta a la terapia y otros factores inherentes a la enfermedad y al \\ paciente. \\ Se presenta el caso de dos niños diagnosticados en el año 2009. El primero fue una niña de ocho \\ años de edad que consultó por palidez cutánea, anemia, leucopenia y blastos en el hemograma. El \\ segundo caso fue un niño de 10 años que consultó por dolor abdominal, que tuvo que ser intervenido \\ por peritonitis secundaria a una apendicitis y en quien se demostró la presencia de blastos en sangre \\ periférica a las 48 horas del posoperatorio. La morfología celular y la citometría de flujo confirmaron, en \\ el primer caso, leucemia linfoide aguda bilineal y, en el segundo caso, leucemia bifenotípica.
}

Palabras clave: leucemias agudas, inmunofenotipo.

\section{Biphenotypic leukemias diagnosis by flow cytometry}

Biphenotypic leukemias are rare in children and represent less than $5 \%$ of all histological subtypes of leukemias. Diagnosis requires careful assessment by cell morphology, genetics, and flow cytometry. The treatment goal is to eliminate the predominant clone, although there is no conclusive data about complete remission. Chemotherapy has shown better results than bone marrow transplant. Survival is influenced by therapy response and other factors inherent to the disease and the patient.

Two patients diagnosed in 2009 are presented. The first patient was an 8 year-old girl who consulted for skin pallor, anemia, leukopenia, and presence of blasts in the white blood count. The second patient was a 10 year-old boy who complained of abdominal pain as result of peritonitis secondary to an acute appendicitis. The presence of blasts in peripheral blood was documented 48 hours after surgery. Cell morphology and flow cytometry confirmed the first case as acute lymphocytic leukemia, and the second as bilinear biphenotypic leukemia.

Key words: acute leukemias, immunophenotyping.

\section{Caso 1}

Se trata de una paciente de sexo femenino de ocho años de edad conocida por el servicio de hematooncología pediátrica desde julio de 2009. Ingresó al servicio por presentar anemia, sin adenopatías ni hepatoesplenomegalia. No se detectó masa de mediastino ni infiltración del sistema nervioso central.

El recuento de leucocitos al momento del diagnóstico fue de 2.100 células/ $\mu$, con $74 \%$ de

\section{Correspondencia:}

Martha Patricia Vizcaíno, Centro Javeriano de Oncología, Pontificia Universidad Javeriana, Carrera $7^{a}$ № 40-62, Edificio Santacoloma, Bogotá, D.C., Colombia

Teléfonos: 320 8320, extensión 4701 y 5946161

marvizval@yahoo.com linfocitos, 5,8 $\mathrm{g} / \mathrm{dl}$ de hemoglobina y recuento de plaquetas de $116.000 / \mathrm{mm}^{3}$.

Mediante el estudio morfológico e inmunofenotípico por citometría de flujo del aspirado de médula ósea, se confirmó el diagnóstico de leucemia linfoide aguda de linaje B de fenotipo común. El análisis inmunofenotípico reportó infiltración de la médula ósea por $73 \%$ de linfoblastos con expresión de los marcadores de linaje B inmaduro: CD45, CD34, CD19, HLA-DR, CD79a (citoplasma) y CD38, y, además, con expresión parcial de CD10 y TdT, y expresión aberrante del marcador de linaje mieloide CD33 (figura 1).

El análisis genético de los hemoblastos reportó negatividad para el cromosoma Filadelfia y para 

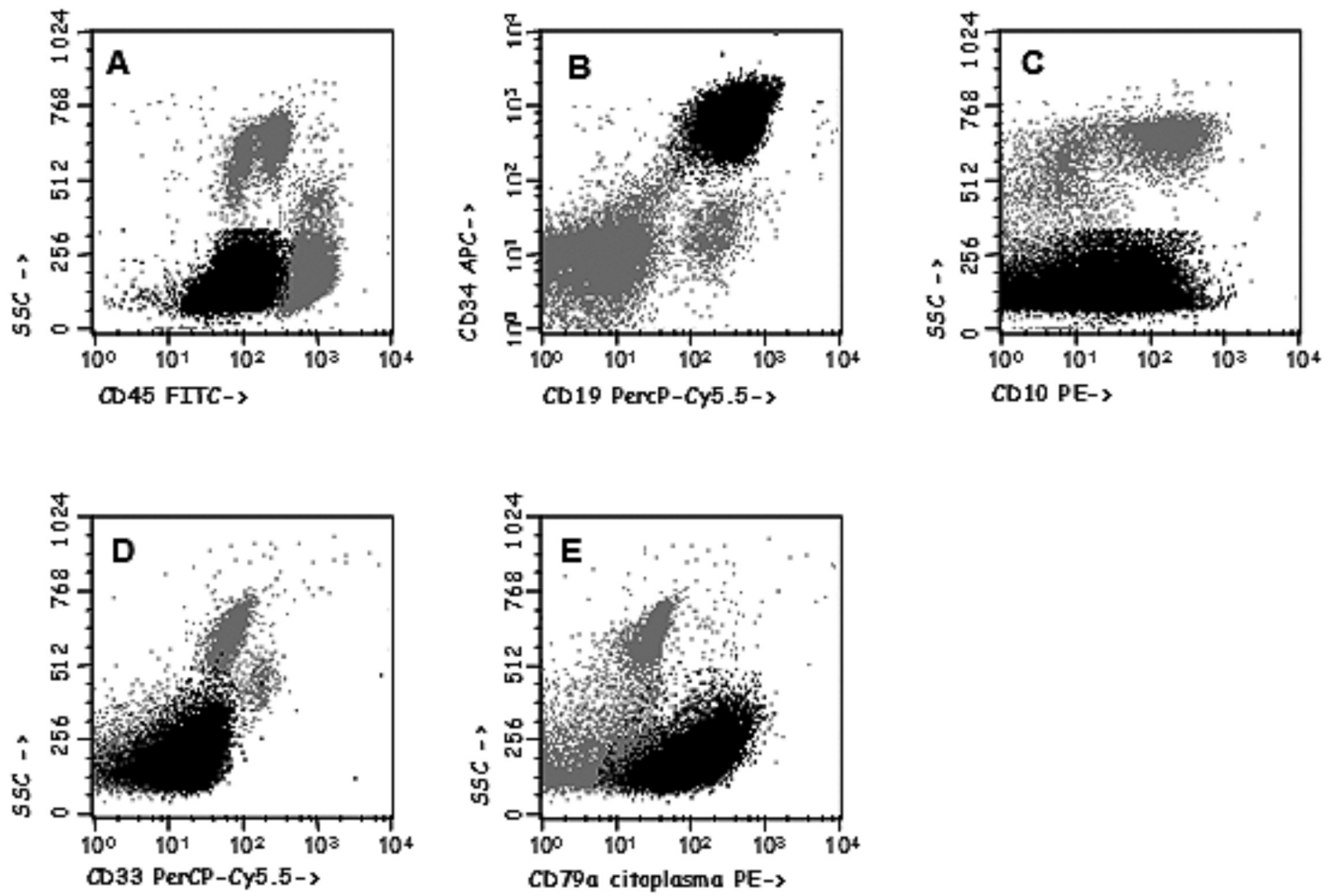

Figura 1. Análisis inmunofenotípico por citometría de flujo de aspirado de médula ósea en el que se detecta infiltración de $73 \%$ de linfoblastos de LLA-B (eventos de color negro) CD45+ (panel A), CD19+/CD34+ (panel B), CD10+ (panel C), con expresión parcial de CD33 (panel D) y CD79a de citoplasma positivos (panel E).

reordenamientos en 11q23 (localización del gen MLL, mixed lineage leukemia).

Se inició tratamiento con prednisona con el protocolo del grupo BFM (Berlin-Frankfurt-Munster). Al día $8^{\circ}$ de tratamiento con esteroides, se encontró aumento de los leucocitos de $85.000 / \mu l$ con $58 \%$ de blastos y aumento de los monocitos iniciales de $4,3 \%$ en el momento del diagnóstico a $67 \%$.

Se analizaron nuevamente la médula ósea y la sangre periférica, por citometría de flujo. En el aspirado de médula ósea y en la muestra de sangre periférica, se encontraron $21 \%$ y $75 \%$ de monocitos, respectivamente, con expresión de los marcadores típicos de este linaje: CD45, CD64, CD14, CD33, CD34, HLA-DR, CD13, CD11b y CD15, con expresión aberrante del marcador del linfocitos B CD19, linfocitos NK CD57 y linfocitos T CD2 (figura 2).

Esto confirmó el diagnóstico de leucemia mixta bilineal versus cambio de linaje. La paciente continuó con el protocolo instaurado y alcanzó la remisión completa al final de la inducción y se encuentra en remisión por más de 9 meses.

\section{Caso 2}

Se trata de un paciente de sexo masculino de nueve años de edad, que consultó por un cuadro clínico de dolor abdominal, en quien se confirmó apendicitis aguda con peritonitis.

En un hemograma posterior al del cuadro agudo, se encontró anemia (8,8 $\mathrm{g} / \mathrm{dl}$ de hemoglobina), $95 \%$ de linfocitosis y $6 \%$ de linfocitos reactivos. El análisis del aspirado de médula ósea mediante citometría de flujo mostró infiltración de $79 \%$ por blastos, los cuales presentaban tamaño mediano con expresión de los marcadores CD45, CD34, CD123 (IL-3R), HLA-DR, en conjunto con marcadores de linaje mieloide CD15, CD33 y CD117, y con expresión parcial de mieloperoxidasa. Además, expresaban los marcadores de linaje linfoide T CD2 y TdT, con negatividad para marcadores de monocitos CD64, CD14 y CD13, de linfocitos B CD19, CD10 y CD20 $y$ linfocitos NK CD56 (figura 3).

Con estos resultados se confirmó el diagnóstico de leucemia mixta. El estudiogenético para cromosoma Filadelfia y reordenamientos en 11q23 (MLL) fue negativo. Se inició terapia con el protocolo de 

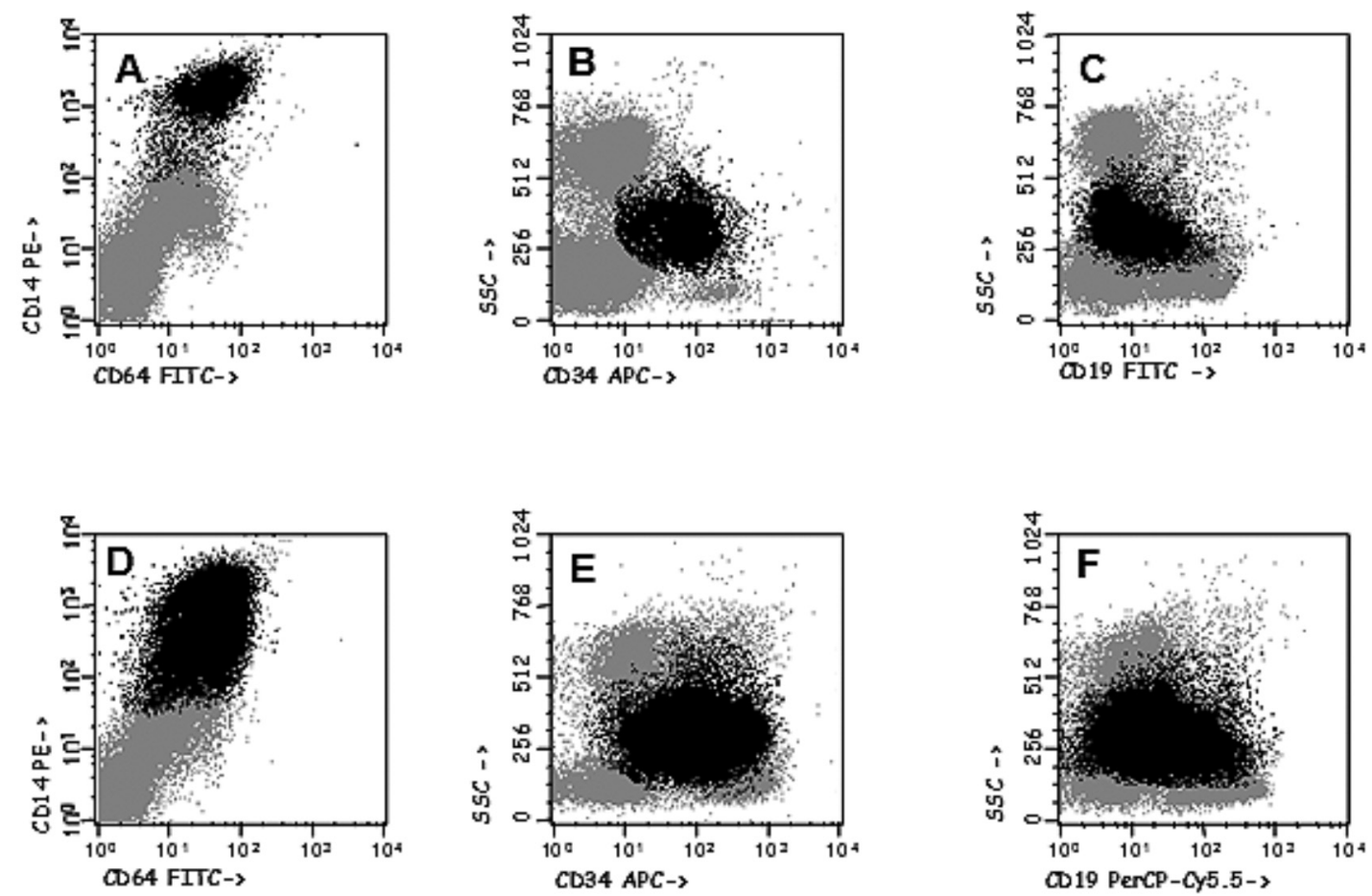

Figura 2. Análisis inmunofenotípico por citometría en aspirado de médula ósea $(\mathrm{MO})$ (paneles $\mathrm{A}-\mathrm{C})$ y de sangre periférica (SP) (paneles D-F) en los que se detectan monocitos CD64+/CD14+ (paneles A y D) con expresión de CD34 (paneles B y E) y expresión aberrante de CD19 (paneles C y F).
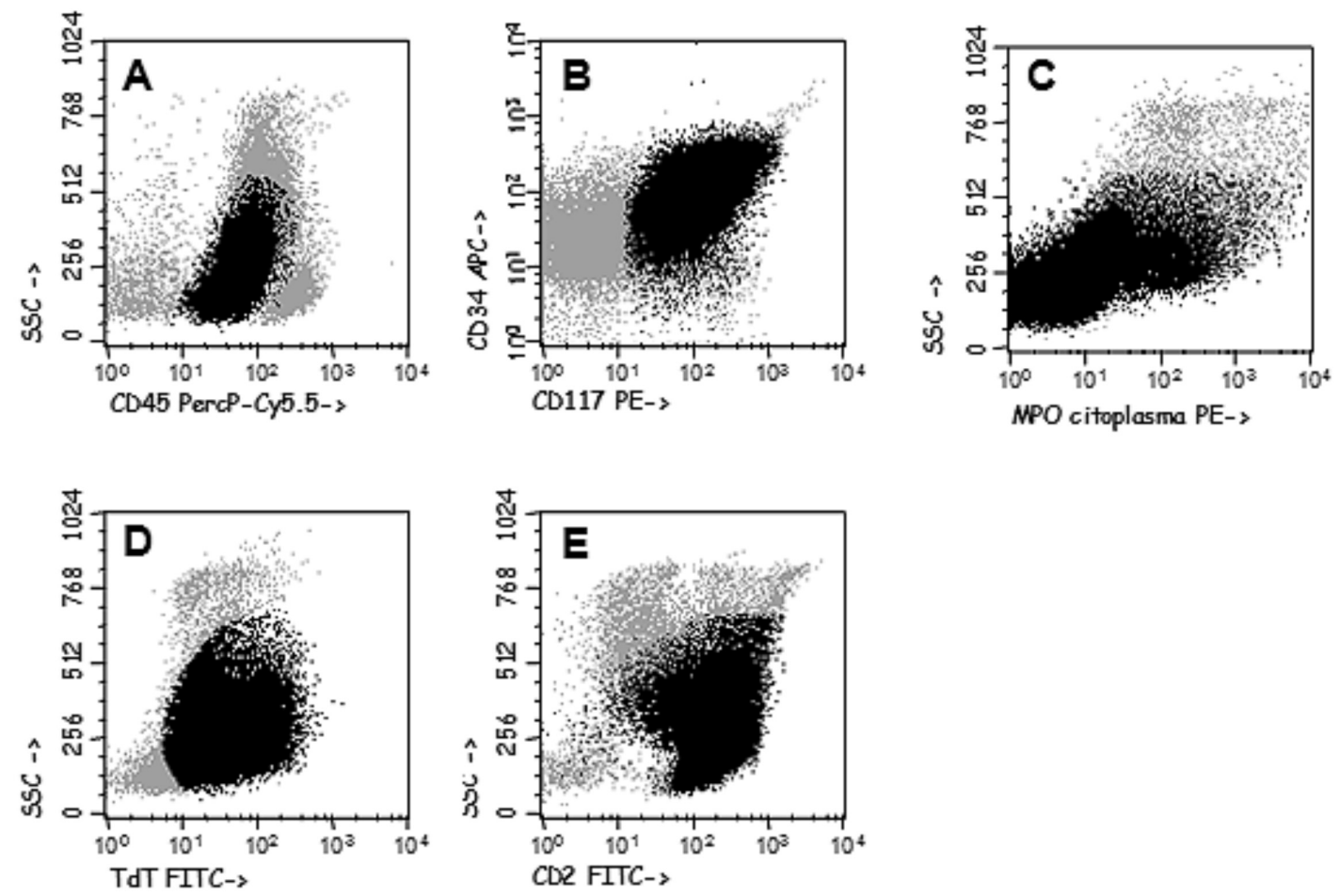

Figura 3. Análisis inmunofenotípico por citometría en aspirado de médula ósea $(\mathrm{MO})$ con infiltración de 78\% de blastos con expresión de CD45 (panel A), CD34 y CD117 (panel B), expresión parcial de mieloperoxidasa (MPO) (panel C), y expresión de marcadores de linaje linfoide como TdT (panel D) y CD2 (panel E). 
leucemia mieloide aguda, basado en el protocolo BFM, con respuesta parcial al finalizar la inducción y remisión completa luego de HAMt (high doses aracytin and mitoxantrone). Actualmente, continúa con quimioterapia y en remisión completa.

\section{Discusión}

La leucemia bifenotípica aguda se define como una leucemia en la que la población de blastos expresa simultáneamente marcadores de linaje mieloide y linfoide $(T \circ B)$. Este tipo de leucemia es raro, con una incidencia aproximada de $5 \%$, y es más frecuente en el sexo masculino y en adultos $(1,2)$. Esta amplia variación puede atribuirse a una serie de razones, como la falta de coherencia de los criterios diagnósticos, el uso de un grupo limitado de anticuerpos en los paneles inmunofenotípicos en los diferentes laboratorios, los cuales no incorporan marcadores citoplasmáticos en el panel principal, y el uso de anticuerpos no específicos de linaje.

Entre las posibles causas del desarrollo de este subtipo de leucemia, se ha descrito un bloqueo madurativo de las células y la pérdida de control de la proliferación de la célula precursora hematopoyética pluripotencial. Se propone que dichos cambios son consecuencia de alteraciones genéticas en la población celular que adquiere un potencial de diferenciación hacia cualquier linaje en una etapa primitiva de la hematopoyesis normal (1).

Esta entidad ha sido previamente clasificada como leucemia mixta o híbrida, y se representa como leucemia mieloide aguda con marcador linfoide (LMA-Ly+) y leucemia linfoide aguda con marcador mieloide (LMA-My) $(1,3,4)$.

Se han descrito cuatro subtipos principales de leucemia bifenotípica aguda, basados en un sistema de puntuación que incluye: B-mieloide, T-mieloide, $B$ y $T$-linfoide, y la leucemia de tres linajes.

La Organización Mundial de la Salud (OMS) recomienda la clasificación del grupo europeo (European Group for the Immunological Characterization of Leukemias, EGIL) para la caracterización inmunológica de las leucemias agudas $(6,7)$. Este sistema de clasificación tiene por objeto distinguir las leucemias bifenotípicas agudas (LMA-Ly+ o LMA-My) de las leucemias agudas que presentan expresión aberrante de marcadores de otros linajes (infidelidad de línea).

Se ha descrito que, entre las leucemias linfoides agudas, la frecuencia de leucemia bifenotípica aguda (LLA-My+) es de 5\%, aproximadamente, y, entre las leucemias mieloides agudas, la frecuencia de leucemia bifenotípica aguda (LMA-Ly+) es de alrededor de $15 \%$ a $20 \%$ de los casos.

La clasificación EGIL se basa en un sistema de puntuación con varios marcadores a los que se les asigna una puntuación de 2, 1 o 0,5 en función de su especificidad para el linaje mieloide o linfoide $(1,6)$; de esta manera, una leucemia que exprese marcadores mieloides con un valor total superior a 2 y marcadores linfoides con valor superior a 1, podría considerarse bifenotípica (1). Entre los marcadores analizados en el sistema de puntuación, se considera la positividad a nivel del citoplasma de los marcadores específicos de linaje mieloide, como mieloperoxidasa, marcadores específicos de linaje B CD79a, CD22 e IgM y marcadores específicos de linaje T como CD3. Los casos de leucemia bifenotípica aguda con marcadores de línea $T$ y $B$ o trilineales (presencia de marcadores mieloides, $\mathrm{B}$ y $\mathrm{T}$ ) son muy raros (1).

Otros criterios útiles para el diagnóstico de leucemia bifenotípica aguda, son los criterios del Saint Jude Children's Research Hospital, definidos por Campana et al. en 2009 (5), que, también, se basan en la expresión de dos marcadores de linaje específico, es decir, expresión citoplasmática de mieloperoxidasa para linaje mieloide, CD3 para linaje $T$, y CD79a y CD22 para linaje $B(5,8,9)$.

En cuanto a los hallazgos de laboratorio, la morfología de los blastos en las leucemias bifenotípicas agudas no es coherente, ya que los blastos pueden mostrar características asociadas a diferenciación mieloide, como la presencia de gránulos azurofílicos o bastones de Auer, o características asociadas a diferenciación linfoide. En algunos casos, se observan blastos que comparten características morfológicas linfoides y mieloides. El tipo mixto polimorfo se asocia comúnmente con leucemia bifenotípica aguda $\mathrm{T}$ mieloide, mientras que la morfología monomórfica descrita en el consenso French American British (FAB) como ALL-L1/L2 se asocia con leucemia bifenotípica aguda $B$ mieloide (1). Los análisis citogenéticos muestran que no hay ninguna anormalidad cromosómica única en la leucemia bifenotípica aguda. Sin embargo, los estudios actuales han demostrado que las anormalidades estructurales son comunes y hay una alta incidencia de casos positivos para el cromosoma Filadelfia $(\mathrm{Ph}+)$ y reordenamientos a nivel de 11q23 y 14q32 $(8,10,11)$.

Los datos sobre los protocolos de tratamiento y los resultados del tratamiento en pacientes con 
leucemia bifenotípica aguda, son limitados. No hay acuerdo sobre si la terapia de inducción debe dirigirse hacia la leucemia linfoide, la mieloide 0 ambas, o si debe seguirse de trasplante de células hematopoyéticas (8). Los protocolos de tratamiento varían según la elección del oncólogo tratante.

En cuanto al pronóstico, se ha descrito que la edad al momento del diagnóstico, y la expresión de HLADR y de CD34, se asocian con un pobre resultado del tratamiento (12), en conjunto con la presencia del cromosoma $\mathrm{Ph}$ y con reordenamientos del gen $M L L$. Además, entre las leucemias bifenotípicas agudas, las de origen linfoide se asocian con mejor pronóstico $(5,8)$.

\section{Referencias}

1. Matutes E, Morilla R, Farahat N, Carbonell F, Swansbury $\mathbf{J}$, Dyer M, et al. Definition of acute biphenotypic leukemia. Haematologica. 1997;82:64-6.

2. Owaidah TM, Al-Beihany A, Iqbal MA, Elkum N, Roberts GT. Cytogenetics, molecular and ultrastructural characteristics of biphenotypic acute leukemia identified by the EGIL scoring system. Leukemia. 2006;20:620-6.

3. Sobol RE, Mick R, Royston I, Davey FR, Ellison RR, Newman R, et al. Clinical importance of myeloid antigen expression in adult acute lymploblastic leukemia. $\mathrm{N}$ Eng $\mathrm{J}$ Med. 1987;316:1111-7.

4. Pui Ch, Raimondi S, Head D, Schell MJ, Rivera G, Mirro $\mathbf{J}$, et al. Characterization of childhood acute leukemia with multiple myeloid and lymphoid markers and diagnosis at relapse. Blood. 1991;78:1327-37.
5. Rubnitz JE, Onciu M, Pounds S, Shurtleff S, Cao X, Raimondi SC, et al. Acute mixed lineage leukemia in children: the experience of St. Jude Children's Research Hospital. Blood. 2009;113:5083-9.

6. Bene MC, Castoldi G, Knapp W, Ludwig WD, Matutes E, Orfao A, et al. Proposals for the immunological classification of acute leukemias. European Group for the Immunological Characterization of Leukemias (EGIL). Leukemia. 1995;9:1783-6.

7. Borowitz MJ, Bene MC, Harris NL, Porwit A, Matutes E. Acute leukemias of ambiguous lineage. In: Swerdlow $\mathrm{SH}$, Campo E, Harris NL, Jaffe ES, Pileri SA, Stein H, et al., editors. WHO Classification of Tumours of Haematopoietic and Lymphoid Tissues. Lyons: IARC Press; 2008. p. 150-5.

8. Al-Seraihy AS, Owaidah TM, Ayas M, El-Solh H, AlMahr M, Al-Ahmari A, et al. Clinical characteristics and outcome of children with biphenotypic acute leukemia. Haematologica. 2009;94:1682-90.

9. Pui CH, Sandlund JT, Pei D, Campana D, Rivera GK, Ribeiro RC, et al. Improved outcome for children with acute lymphoblastic leukemia: results of Total Therapy Study XIIIB at St. Jude Children's Research Hospital. Blood. 2004;104:2690-6.

10. Cuneo A, Ferrant A, Michaux JL, Demuynck H, Boogaerts M, Louwagie A, et al. Philadelphia chromosome positive acute myeloid leukemia: cytoimmunologic and cytogenetic features. Haematologica. 1996;81:423-7.

11. Carbonell F, Swansbury J, Min T, Matutes E, Farahat N, Buccheri V, et al. Cytogenetic findings in acute biphenotypic leukaemia. Leukemia. 1996;10:1283-7.

12. Gujral S, Polampalli S, Badrinath Y, Kumar A, Subramanian PG, Raje G, et al. Clinico-hematological profile in biphenotypic acute leukemia. Indian $\mathrm{J}$ Cancer. 2009;46:160-8. 